\title{
Electrospinning and Electrospun Nanofibers
}

\author{
Lefayet Sultan Lipol, Md. Moshiur Rahman \\ University of Borås, Borås, Sweden \\ Email: lefayetbd@gmail.com
}

Received 31 December 2015; accepted 12 April 2016; published 15 April 2016

Copyright (C) 2016 by authors and Scientific Research Publishing Inc.

This work is licensed under the Creative Commons Attribution International License (CC BY).

http://creativecommons.org/licenses/by/4.0/

(c) (i) Open Access

\begin{abstract}
Electro-spinning is a very modern process which can be used in various purposes. We did this experimental work at Swerea IVF in Sweden during M. Sc in Textile Technology programme at University of Borås. We should especially thank our supervisor-Anna Thorvaldsson and course teacher-Ioannis S. Chronakis. In this report, we have tried to explain the basic manufacturing techniques of the electrospun nanofiber by the electro-spinning, how one can characterize it by SEM (Scanning Electron Microscopy) and its various applications in the practical field, e.g wound healing, Tissue Engineering Scaffold. The experimental work helped us a lot to gather sufficient knowledge about the electro-spinning process which we wanted to share with all.
\end{abstract}

\section{Keywords}

Nano-Fibers, Tissue Engineering Scaffold, Nano-Collagen, Cartilage and Bones, Bladder and Kidney, SEM (Scanning Electron Microscopy)

\section{Background}

Electro-spinning is a best way of producing fibers in nano and micro size scale. A polymer solution is fed through a syringe with a metal needle. A high voltage is connected between the needle and a grounded collector, creating an electric field in which the fiber is immensely stretched upon its way to collector. The stretching together with evaporation of the solvent renders fibers in nano to micro size to be collected on the collector. Applications of the nanofibers are sound absorption, filters, sensors and biomedicine.

\subsection{Factors Are Affecting Electro-Spinning Process}

Viscosity, pressure of the pump, distance between plate and needle, the electric field, type of fiber and solution, temperature, different kind of plate, drying the fabric after fabrication [3].

\subsection{Key Dimensions}

Polymeric nano-fibers: $50 \mathrm{~nm}$ to 1 micro; blood cell: 5 micro; human hair: 20 - 30 micro [3]. 


\subsection{Remarkable Features}

1) Technological Motivation;

2) High surface area per unit mass;

3) (diam. of $100 \mathrm{~nm}-1000 \mathrm{~m}^{2} / \mathrm{g}$ );

4) Small and specific pore size;

5) Extremely long length (kilometers);

6) $3 \mathrm{D}$ continuous structures (unlike CNT, nanorods);

7) High axial strength combined with extreme flexibility;

8) Mixture of polymers is feasible;

9) Alignment of nanofibers (nanotubes \& nanowires) is feasible;

10) Nanoparticles can be encapsulated;

11) Variety of cross-sectional shapes \& sizes;

12) Various routes for functionalization ( PVD, CVD etc.);

13) Cost effective top-down nanomanufacturing process (compared to bottom up) [3].

\section{The Basic Set Up for Electro-Spinning}

In Figure 1, It consists of three major components: a high voltage power supply, a spinneret (a metalic needle), and a collector (a grounded collector).

The spinneret is connected to a syringe in which the polymer solution (or melt) is hosted. With the use of a syringe pump, the solution can be fed through the spinneret at a constant and controllable rate. When a high voltage (usually in the range of 1 to $30 \mathrm{kV}$ ) is applied, the pendent drop of polymer solution at the nozzle of the spinneret will become highly electrified and the induced charges are evenly distributed over the surface. As a result, the drop will experience two major types of electrostatic forces: the electrostatic repulsion between the surface charges; the coulombic forces exerted by the external electric field.

Under the action of these electrostatic interactions, the liquid drop will be distorted into a conical object commonly known as Taylor cone. Once the strength of the electric field has surpassed a threshold value, the electrostatic force can overcome the surface tension of the polymer solution and thus force the ejection of a liquid jet from the nozzle. The electrified jet then undergoes a stretching and whipping process, leading to the formation of a long and thin thread. As the liquid jet is continuously elongated and the solvent is evaporated, its diameter can be greatly reduced from hundreds of micrometer to as small as tens of micrometers. Attracted (opposite charge) by the grounded collector placed under the spinneret, the charged fiber is often deposited as a randomly oriented, non-woven mat [1].

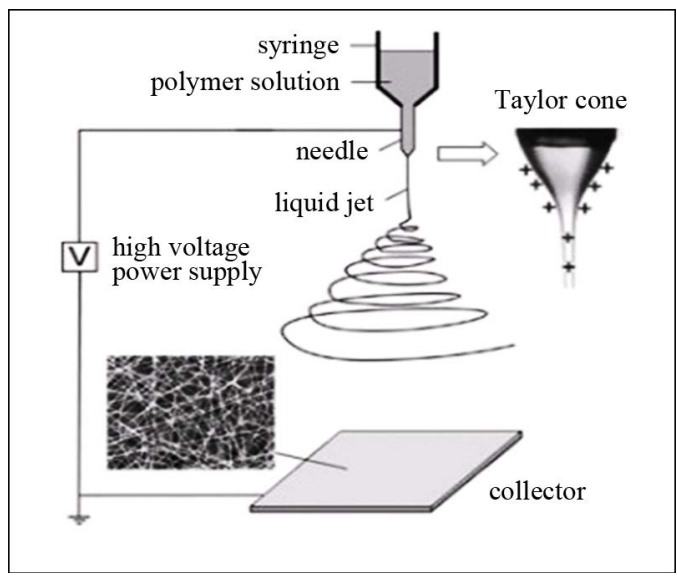

Figure 1. Schematic illustration of the basic set up for the electro-spinning [1].

\subsection{How Does the Fibers Become So Thin}

a) Bending instability so every loop grows larger and larger; 
b) The jet streches and becomes thinner;

c) The jet can be sterched untill the diameter is about $100 \mathrm{~nm}$ [3].

\subsection{Control of Electro-Spun Nanofibers}

a) Control of Morphology and Diameter;

b) Control of Chemical Composition: (Functional Polymers \& Blends, Encapsulation of Functional Nanomaterials, Modification of Nanofibers);

c) Control of Secondary Structures: (Core/Sheath Nanofiber Structures, Hollow Interiors Nanofibers, Porous Nanofiber Structures) [3].

\subsection{Examples of Polymer-Solvents System}

In Table 1, we mention here names of the some polymers and the solvents are used for producing electro-spun nano-fibers.

\begin{tabular}{ccc} 
Table 1. Polymer-solvent system $[3]$ & \\
\hline Sl. No. & Polymers & Solvents \\
\hline $\mathbf{0 1}$ & Nylon 6 and nylon 6,6 & Formic acid \\
$\mathbf{0 2}$ & Polyacrylonitrile & Dimethyl Formaldehyde \\
$\mathbf{0 3}$ & PET & Trifluroacetic acid/Dimethyl chloride \\
$\mathbf{0 4}$ & PVA & Water \\
$\mathbf{0 5}$ & Polystyrene & DMF/Toluene \\
$\mathbf{0 6}$ & Nylon-6-co-polyamide & Formic acid \\
$\mathbf{0 7}$ & Polybenzimidazole & Dimethyl acetamide \\
$\mathbf{0 8}$ & Polyramide & Sulfuric acid \\
$\mathbf{0 9}$ & Polyamides & Phenol \\
\hline
\end{tabular}

\subsection{Nanofiber with Core/Sheath Structure}

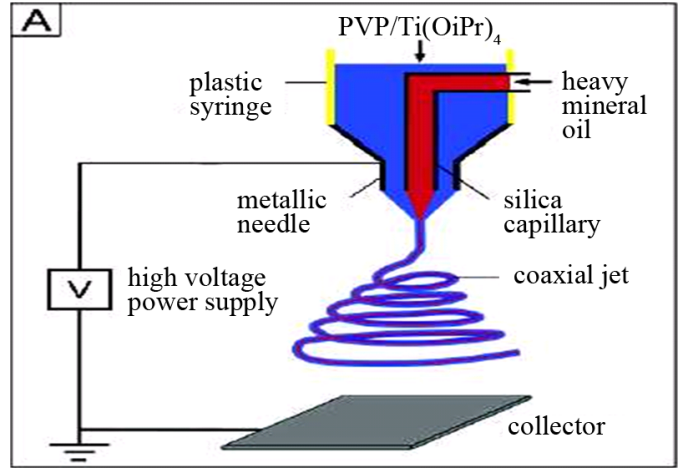

Figure 2. Nanofibers with core/sheath structure [1].

From the above shown process in Figure 2, we can make the nanofibers with hollow interiors, nanofibers with porous structures.

The spinneret has two coaxial capillaries. The silica capillary contains mineral oil while the solution in the plastic syringe is containing poly (vinyl pyrrolidone)/(PVP) and titanium tetraisopropoxide/Ti( $\mathrm{OiPr})_{4}$ were simultaneously ejected to form a compound jet [1].

\section{Sputtering in SEM}

- In Figure 3, the electro-spun nanofiber with the coating of the gold is used. The gold is used for more visibility 
of the electro-spun nanofibers under Scanning Electron Microscopy (SEM). The fiber is set over a slide before putting under SEM.

- The polymer was used Eudragit (Methacrylic acid) - 15\% with Solvent: 1:1 Ethanol/Formic acid to form the electro-spun nanofibers which was observed under SEM to predict the characteristics.

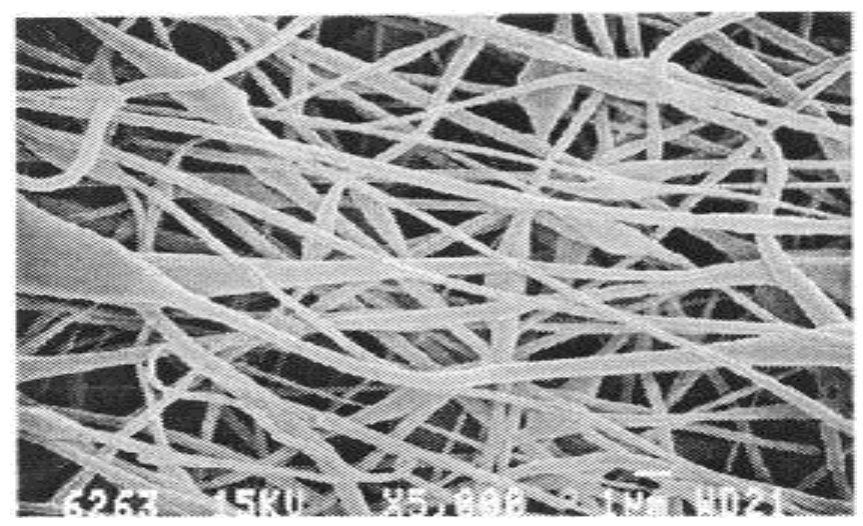

Figure 3. Nanofibers on SEM [2].

We can evaluate the fiber performance by using scanning electron microscopy (SEM) and contact angle measurements. If the contact angle of the fiber surface with liquid is high, the fiber is hydrophobic. Sessile drop can be used as liquid [2].

\section{Application of Nanofibers}

The applications of nanofibers are mentioned in Table 2 (summary of the application of the Nanofibers).

\section{Table 2. Application of nanofibers [3].}

\begin{tabular}{|c|c|c|}
\hline Sl. No. & The area of the applications & Applications \\
\hline 01 & Nanosensors & $\begin{array}{l}\text { Nanoelectronics, optical devices, nanowires, } \\
\text { interconnect. }\end{array}$ \\
\hline 02 & Coating & Protective clothing, sound absorption. \\
\hline 03 & Biomedical applications & $\begin{array}{l}\text { Wound healing, artificial skin, scaffolding, tissue } \\
\text { engineering. }\end{array}$ \\
\hline 04 & Nano filter media & nano membranes \\
\hline 05 & Other industrial applications & Nanocomposites, nanoceramics, nano catalysts \\
\hline
\end{tabular}

\subsection{Wound Healing}

Polyurethane dressing: EVOH electro spun directly onto a human hand. EVOH is just a medical application polymer (Ethylene-vinyl alcohol copolymer) [3].

\subsection{Features of Material Use as Tissue Engineering Scaffold}

Various specific shape for specific applications, porosity of membranes for skin, hydrophillicity, biodegradation property, conductivity, filtration, binding ability, flexibility, tolerate sudden force, cell adhesion and migration. Tengion is one of very few companies making business of tissue engineered products. They produced tissue engineered bladders. Because it is very simple tissue to manufacture, it is easy to biomimic the bladder. Liver is complicated to imitate [2].

\subsubsection{Important Features to Consider for Selecting Scaffold}

It must tolerate certain force; it must have resiliency, high tear tension, elasticity, work of rupture [1]. 


\subsubsection{Basic of Working of a Tissue Engineering Scaffold}

The mixture of the cell and the salt (solution) on nanofibers surface increases attraction power of the cell because of salt and there may be some porosity as well on the cell. Salt gives away the cell in solution. Then these cells attract other cells to adjust with it [3].

\subsubsection{Important Notes}

Nanofiber is used to produce bladder commercially. The bladder is used to pass urine that comes from the kidney [2].

Water + dirt $\rightarrow$ Kidney (pure liquid) $\rightarrow$ Bladder (pass urine).

\subsubsection{Difference between Injection and Implantation}

If we inject something that is injection, making two layers and then insert scaffold is implantation [2].

\subsection{Drug Delivery}

It can be observed porosity or hole in the nanofibers under Scanning Electron Microscopy (Figure 3). One can insert drug in that specific regions. After that one will implant it in the patient's body. Because of porosity, drug can move from the fiber as well as they have hydrophillicity. This is the reason why drugs are dispersed in the blood and in that way the fiber can be used to deliver drug in the fiber. In addition, the fibers are biodegradable so they will not create any problem in the human body.

When doctors make operation of a patient, they need to put medicine on a specific region. This is the reason why they need one carrier to hold the medicine on the exact place. The electrospun nanofibers are used for this purpose. The fibers do not create any problem in the human body in the long run as it is biodegradable [2].

\subsection{Difference between Cartilage and Bones}

In cartilage has no blood vessels but in bone has blood vessels. Cartilage is a soft bone found in ear, nose. Cartilage absorbs the forces that bones applied so the bone can recover to a large extent if it is forced by external objects.

We can make a hole over the bones or cartilage as well as insert the blood in holes, coagulate the blood. And this blood attracts other cells and helps to the cells grow over it. In a word, blood work like a scaffold there [2].

\subsubsection{Nanocollagen}

It is used to replace damaged blood vessels [2].

\subsection{Air Filtration}

Air filtration by nanofiber is cheaper than commercial air filtration process as well as it is more effective.

Moreover, nanofibers can be used for the liquid filtration and to for the acoustic insulation [4] [5].

\section{Discussion}

Electro-spun fibers are produced according to Figure 1. It is necessary to use polymer and solvent together for producing nanofibers. When a high voltage is applied, the pendent drop of polymer solution at the nozzle of the spinneret will become highly electrified and the induced charges are evenly distributed over the surface. As a result, the drop will experience two major types of electrostatic forces. Under the action of these electrostatic interactions, the liquid drop will be distorted into Taylor cone. When the fibers will be formed, the solvent will be evaporated to the environment. The electro-spun nanofibers with porous structure can be formed according to Figure 2. We can insert required substances in the porous structure. The characteristics of the electro-spun nanofibers can be identified by Scanning Electron Microscopy. These fibers can be used for various purposes. For instance-wound healing, tissue engineering scaffold, drug delivery, air filtration etc.

\section{Conclusion}

Electro-spinning is the way to produce nanofibers which have various prolific applications. From this research 
work, we have understood the manufacturing process of the electro-spun nanofibers and its characteristics and applications. This is the reason why we hope that the practical experience should help us in the future.

\section{References}

[1] Li, D. and Xia, Y.N. (2004) Electro-Spinning of Nanofibers: Reinventing the Wheel. Advanced Materials.

[2] (2009) Laboration Work at Swerea IVF (Supervisor-Anna Thorvaldsson). 5 March 2009, M. Sc in Textile Technology Program at University of Boras, Textile Fiber Material Course.

[3] (2009) Ioannis, S., Chronakis, Swerea IVF, M. Sc in Textile Technology Program, University of Boras, Lecture Materials of Textile Fiber Material Course.

[4] (2015) Elmarco Nano for Life. Date: 2015-07-14. http://www.elmarco.com/application-areas/air-filtration/

[5] (2015) Electro-Spun Nano-Fibers for Air Filtration. Date: 2015-07-14. http://www.sciencedirect.com/science/article/pii/S1877705813018092

\section{About Authors}

Author-1: I have completed B. Sc in Textile Technology from College of Textile Technology, Tejgaon, Dhaka-1208 under Dhaka University. My specialization was in wet processing technology.

In Sweden, I have completed three M. Sc degrees from Boras University. The degrees are-Textile Engineering, Applied Textile Management and Industrial engineering. I have published six international journals and 3 magazine papers. I worked in Sweden as Researcher at SP Technical Research Institute of Sweden. Presently, I work as Lecturer and Program coordinator of the Textile Engineering department at Fareast International University, Bangladesh.

Author-2: I have completed B. Sc in Textile Technology from Bangladesh University of Textiles (BUTEX), Tejgaon, Dhaka-1208. My specialization was in yarn manufacturing technology and obtained the 1st class.

I started my career as a Trainee Merchandiser at COTTON GROUP and then switched to EPYLLION GROUP as an Assistant Merchandiser. Then I worked at Matin Spinning Mills Ltd. (a sister concern of DBL GROUP) as an Executive in the department of Quality Control. Finally I joined at Northern University Bangladesh as a Lecturer in the department of textile engineering. At present, I am a permanent employee and attend at different programs arranged by this university. 